\title{
LA FILOSOFÍA DE LA BIOLOGÍA Y LOS ESTUDIOS DE GÉNERO. UNA SIMBIOSIS DEMORADA
}

\author{
FABrizzio GuERrero MC MANus \\ Centro de Investigaciones Interdisciplinarias \\ en Ciencias y Humanidades \\ Universidad Nacional Autónoma de México \\ FabrizzioMc@gmail.com
}

RESUMEN: Este texto busca generar un espacio de interlocución entre filósofos de la biología y estudiosos del género. En el marco del feminismo analítico ofrece dos ejemplos concretos que buscan ilustrar la fecundidad de dicho encuentro. Por un lado, se examina la acusación de esencialismo que generalmente se esgrime en contra de la biología y se le replantea a la luz de la filosofía de la taxonomía. Por otro lado, se analiza la estructura de las explicaciones biológicas del género y la sexualidad a la luz de las llamadas falacias ontogenética y filogenética.

PALABRAS CLAVE: feminismo analítico, esencialismo metodológico, falacia filogenética, falacia ontogenética, agnotología

SUMMARY: This text seeks to promote a more fruitful collaboration between philosophers of biology and gender specialists. Framed under the Analytical Feminist perspective, it offers two examples that emphasize the fecundity of this effort. On the one hand, the accusations of essentialism usually endorsed against biological approaches to gender and sexuality are revisited and reframed in light of the philosophy of taxonomy. On the other hand, the structure of the biological explanations of gender and sexuality is discussed taking into account the ontogeny and phylogeny fallacies.

KEY WORDS: analytical feminism, methodological essentialism, phylogeny fallacy, ontogeny fallacy, agnotology

El objetivo de este ensayo es hacer una invitación tanto a los filósofos de la biología como a los especialistas en temas de género para que consideren la posibilidad de construir espacios de interlocución entre sus comunidades. Asimismo, busco evidenciar un conjunto de conexiones que sirvan de puente entre ambas disciplinas. Por último, con esta contribución aspiro a construir dichas conexiones al interior de una comunidad hispanohablante en la cual muy poco se ha dicho sobre cómo conectar ambos saberes.

En este sentido, he calificado la relación que actualmente existe entre ambos campos como "una simbiosis demorada" precisamente para enfatizar que hoy día esta interlocución es prácticamente nula, y que a pesar de esto, podría conducir a una redefinición de algunas de las controversias que le son comunes a estas áreas — como la dis- 
cusión naturaleza $v s$. crianza-, de tal suerte que ambas disciplinas terminen por convertirse en simbiontes mutuamente dependientes.

Desde luego, no quiero que se entienda que no han habido esfuerzos por combinarlas, de hecho, sí los han habido, sobre todo en el mundo anglosajón con el llamado feminismo analítico (Garry 2012), pero estos esfuerzos no han terminado por consolidar un espacio común entre estos saberes. ${ }^{l}$ Es más, dichos esfuerzos no son exclusivos de las filósofas mencionadas, ya que al interior mismo de la biología se han gestado importantes posturas feministas que no han recibido mucha atención dentro del grueso de los estudios de género (e.g., Hrdy 1999, Poiani 2010, Roughgarden 2004, 2009, y Zuk 2002).

Sospecho que esto se debe a que la distinción entre filosofía analítica vs. filosofía continental no solamente suele colocar en lados distintos a estos dos cuerpos disciplinares, sino a que ha terminado por poner una barrera al interior de los estudios de género y sexualidad entre aquellos sectores que serían más afines a una tradición continental y aquellos más afines a una tradición liberal cercana en sus formas de argumentación a la tradición analítica. A modo de ejemplo, piénsese en la diversidad de posiciones que informan a los estudios de género y sexualidad y que tienen su origen en una gran variedad de feminismos de corte marxista (e.g., Silvia Federeci 2004), de corte posestructuralista y con influencias del psicoanálisis lacaniano (e.g., Luce Irigaray 1985, Julia Kristeva 1982, Judith Butler 1993, y Beatriz Preciado 2002) o de corte liberal y con inspiración rawlsiana (e.g., Nussbaum 2010, 2012).

Esta escisión puede observarse en los diferentes diagnósticos y propuestas normativas que provienen de los diversos proyectos feministas de algunas de las autoras antes mencionadas así como también en la forma en la que se critican fuertemente unas a otras. ${ }^{2}$ Desde luego, en este sentido, el feminismo y los estudios de género y sexualidad son parte de un enfrentamiento histórico mucho más extenso ${ }^{3}$ que ha tenido momentos de gran notoriedad (e.g., los debates Chomsky-Foucault (2006) y Derrida-Searle (Navarro Reyes 2010)).

Sea como fuere, tengo la creencia de que tanto unos como otros tienen mucho que ganar con esta interlocución aunque exija vencer la

${ }^{1}$ Véase, por ejemplo, Fausto-Sterling 2000, Haraway 1989, Keller 1997, Lloyd 2005, Longino 2002, y Oyama 2000.

${ }^{2}$ Por ejemplo, Nussbaum (1999) critica y cuestiona las formas de argumentación y las propuestas de acción política de Butler.

${ }^{3}$ Para una lectura de cómo inició dicha escisión véase, por ejemplo, Friedman 2000. 
desconfianza creada en ambos lados de esta división. Para mostrar el porqué de mi optimismo examinaré un conjunto de temas asociados a la dicotomía naturaleza vs. crianza. Dicha dicotomía es el campo dentro del cual se llevan a cabo gran parte de los enfrentamientos en torno a la "naturaleza humana" (Dupré 1993, 2002) y es, por lo tanto, un lugar privilegiado para examinar las posibles sinergías.

Básicamente, en una primera sección de este ensayo analizaré algunas discusiones de tipo ontológico acerca del esencialismo en biología. En esta sección mostraré cómo la filosofía de la biología nos ayuda a comprender exactamente qué se está entendiendo por esencialismo en biología a la luz de estos debates.

Ahora bien, en una segunda sección me concentraré en temas de corte epistemológico para examinar los límites de las explicaciones biologicistas así como también los puntos de coincidencia que hay entre algunos filósofos de la biología y los especialistas sobre género y sexualidad. Por último, concluiré este texto señalando un conjunto de posibles zonas de contacto que valdría la pena explorar en un futuro.

\section{Ontologías}

Quizá la acusación más comentada en la historia de los estudios de género y sexualidad es la famosísima afirmación del carácter esencialista de las ciencias biomédicas y biológicas en lo que al género y la sexualidad respecta (DeLamater y Shibley 1998). Dicha acusación es, empero, una instancia del argumento antiesencialista que los constructivistas sociales esgrimen al interior de los debates para saber si hay algo así como una naturaleza humana en temas tales como la raza, la inteligencia, o algún otro atributo humano presuntamente pancultural — es decir, presente en toda cultura- (Dupré 1993, 2002).

Con frecuencia esta afirmación, al menos en el caso de los estudios de género y sexualidad, se traduce en una acusación a la biología y a la biomedicina de postular esencias o naturalezas transhistóricas, panculturales y definitorias no sólo de lo masculino y de lo femenino en tanto corporalidades, sino también al nivel mismo de lo psíquico (deseos, capacidades cognitivas, etc.). Desafortunadamente, como he expresado en otra parte (Mc Manus 2012), esta forma de construir el argumento antiesencialista a los biólogos y a los filósofos de la biología les resulta completamente extraño por una razón más o menos evidente: la evolución. 
Y es que el impacto de la Teoría Evolutiva, tanto en la filosofía (e.g., los pragmatistas estadounidenses) como en la biología, supone un abandono de la idea misma del fijismo y la inmutabilidad, en general, y del fijismo de especies, en particular. Más aún, a raíz de los famosos ensayos de Ghiselin (1997) y Hull (1965), ha comenzado una discusión en torno a la metafísica de las especies que realza su historicidad y cuestiona su carácter de clases transhistóricas (Boyd 1999). ${ }^{4}$

Así pues, a nivel ontológico surge claramente una pregunta de interés para todos los involucrados. ¿Es la biología esencialista? Los constructivistas sociales dicen que sí, mientras que los biólogos y los filósofos de la biología, por otro lado, han luchado en contra de un esencialismo de las especies y resulta curioso que ahora se les acuse de esencialistas incluso si reconocemos que hay muchos partidarios de interpretaciones biologicistas de la sexualidad y del género. Pero, entonces, quién tiene la razón.

A mi modo de ver, la tarea del filósofo de la biología debería consistir justamente en tratar de comprender la naturaleza del debate antes de tomar partido. En ese sentido yo comenzaría con dos precisiones. Por un lado, sería importante lograr que los involucrados se den cuenta de que hay más de una manera de construir el esencialismo. Por otro lado, y precisamente porque hay más de una manera de hacerlo - y existe más de un ámbito en el cual se puede ser esencialista, como explicaré inmediatamente - es que se podría ser esencialista bajo cierta interpretación mas no bajo otra.

De cualquier manera, la noción de homología es probablemente un punto de inicio fundamental para esclarecer la naturaleza del debate acerca de si la biología postula alguna clase de esencialismo. Esto es por la siguiente razón: cuando se afirma que la identidad de género o la orientación sexual de un sujeto puede describirse con un mismo aparato categorial más allá de su contexto histórico-social implícitamente se invoca ya la oposición tipo/instancia — type vs. token-, pues se considera que la identidad de género o la orientación sexual son propiedades universalizables que simplemente se ejemplifican en compañía de otras propiedades en individuos concretos; sin duda lo anterior hace posible una comparación entre culturas y, con el tiempo, entre especies. Sin embargo, esto conllevará una construcción en cierto sentido esencialista de los rasgos fenotípicos.

Demostrarlo no es tarea fácil. No obstante, quiero ofrecer un breve argumento de por qué sostengo esto. Primero, a modo de ejemplo,

${ }^{4}$ Véase también Rieppel 2009. 
quiero traer a cuenta cómo se suelen equiparar diversas conductas en diversas culturas. Pensemos en el modo en el cual los paidika griegos, los sodomitas, los cuilloni aztecas y los modernos homosexuales son todos ellos considerados distintos ejemplos de un mismo tipo de conductas: la homosexualidad (Foucault 1977, Guerrero Mc Manus 2013, y Mc Manus 2012). Segundo, esto no sólo sucede entre culturas, también entre especies como cuando las conductas de diversos organismos se ponen como ejemplos de homosexualidad, bisexualidad, travestismo o poligamia (Bagemihl 1999, y Roughgarden 2004); sin embargo, aquí nuevamente está operando la distinción tipo/instancia que logra que conductas diferentes parezcan ser lo mismo.

Anteriormente he afirmado que esta estrategia de construcción de la sexualidad en tanto caracteres fenotípicos es uno de los rasgos estructurales de las explicaciones biológicas de la homosexualidad (Mc Manus 2012), y ahora sostengo que de hecho es un atributo más general y propio de las explicaciones biológicas acerca del género y de la sexualidad. Asimismo, he manifestado que esta estrategia es el resultado de lo que he denominado empirismo sobre el género (Guerrero Mc Manus 2012) que básicamente consiste en construir un espacio conceptual en el cual nuestros sistemas categoriales sobre el género y la sexualidad son representables por medio de distintas dimensiones, cada una de las cuales tiene diversos valores que se corresponden a las distintas propiedades instanciadas.

Siguiendo esta lógica es posible representar a cualquier ser humano, incluso a cualquier animal, se le puede asignar un vector en el cual se especifiquen sus valores particulares para dimensiones independientes como el sexo (función macho vs. función hembra), la morfología (intersexual, masculino, femenino, etc.), la orientación sexual (homosexual, heterosexual, bisexual), la identidad de género (hombre, mujer...), etc. Nótese que en principio es posible introducir más y más dimensiones con más y más valores si fuese necesario distinguir entre especies sexualmente monomórficas o sexualmente dimórficas o, incluso, sexualmente dimórficas, pero con reversión en el papel sexual.

Ahora, ¿cuál sería el problema de llevar a cabo esta taxonomía? Básicamente enfrenta dos problemas. Por un lado, dicho espacio conceptual no recupera la forma en que personas ajenas a dicha taxonomía viven el género y la sexualidad. Esto es, pasa por alto que nuestra forma de catalogar no es universal ${ }^{5} \mathrm{y}$, en este sentido, obvia

${ }^{5}$ Véase el trabajo antropológico de Prieur (2008) de las vestidas en Ciudad 
las dimensiones fenomenológicas que acompañan al género y a la sexualidad porque éstos se corresponderían más con existenciarios que con propiedades (Mc Manus 2012), lo cual explicaría que feministas como Butler (1993) sostengan que el género no es un predicado del sujeto.

Por otro lado, esa pretensión de universalidad apunta justamente al porqué del señalamiento de esencialismo. Metodológicamente hablando, la postulación de dos rasgos como homólogos requiere primero su postulación como semejantes para posteriormente evaluar si dicha semejanza puede concebirse como el producto de la ancestría común; si como resultado de dicha evaluación se afirma que estamos de hecho ante homologías, entonces a los rasgos se les terminarán por plantear evolutivamente como el mismo rasgo a pesar de cualquier posible modificación que hayan sufrido a través de su historia (Mc Manus 2009).

Desafortunadamente, uno de los puntos débiles de estas metodologías es el problema de los rasgos conductuales. Evidentemente la semejanza entre dos conductas no puede considerarse como elemento diagnóstico si las conductas en seres humanos vienen investidas de significación - y erotización-, mientras que esto no parece ser el caso en otros organismos (e.g., no es lo mismo declarar que no se nace mujer sino más bien se llega a serlo, a ser una Drosophila hembra que va zumbando por ahí). $\mathrm{Y}$ es justo en el tema de género y sexualidad que se reconoce que estamos ante una dimensión hipertélica de nuestras conductas (Escobar 2012), es decir, que se rebasa el telos o la función biológica de los órganos.

Así pues, el señalamiento de esencialismo es perfectamente compatible con una biología evolucionista que ha descreído de la fijeza de las especies. Esto es así porque el esencialismo al que nos enfrentamos es fundamentalmente metodológico, ya que emana del uso de la distinción tipo/instancia como una forma de conceptuar a los caracteres fenotípicos conductuales. Y es que dicha distinción es completamente indiferente a las dimensiones fenomenológicas que acompañan a las categorías de género y sexualidad.

Por ello mismo, es allí donde puede apreciarse con mejor claridad el señalamiento de esencialismo. No es un esencialismo que implique la existencia de una esencia que determina propiedades necesarias. Por el contrario, es un esencialismo en el cual se juzga como $l o$ mismo a fenómenos que exhiben diferencias manifiestas al nivel de

Nezahualcóyotl como un ejemplo de cómo género y orientación sexual se viven como una dimensión anastomosada. 
cómo se habita fenomenológicamente un mundo al menos constituido parcialmente por el organismo en cuestión.

Por último, cabe señalar, que hay una petición de principio que hiere fatalmente a este esencialismo metodológico. Y es que la única forma en la cual se puede postular a modo de hipótesis de homología la mismidad de las diferentes construcciones del género y la sexualidad es cuando se pasan por alto las diferencias valorativas, institucionales, contextuales, históricas, lingüísticas, etc., si las hay (generalmente en seres humanos), o la ausencia de éstas, cuando no están (generalmente en animales no humanos) (Guerrero Mc Manus 2013). Curiosamente, esta igualación muchas veces es una heurística que sirve de motor a las aproximaciones biologicistas sobre el género y la sexualidad por el hecho del carácter supuestamente universal y pancultural de estos fenómenos. Es claro que tomar como heurística para buscar una explicación naturalista de un fenómeno que sólo puede constituirse como el mismo fenómeno si se pasa por alto el entramado de significados y prácticas que en cada caso lo acompañan es olvidar, y en este sentido reificar, que ese fenómeno emergió como un mismo fenómeno porque se atendió únicamente aquello que juzgamos hacía idénticos a esos fenómenos dispares.

\section{Epistemologías}

Es común encontrarnos con explicaciones sobre la homosexualidad o sobre la bisexualidad que se basan en la presunción de que éstas son evolutivamente un misterio y que, por lo tanto, tienen que explicarse como disfunciones en mecanismos próximos (Guerrero Mc Manus 2013). Tampoco es inusual encontrar explicaciones sobre rasgos considerados "típicamente" masculinos y femeninos y que presuntamente son resultado de la evolución por selección sexual.

Nótese que en ambos casos existe una falta de simetría. En el primero, la heterosexualidad suele asumirse como algo ya explicado y que no requiere mayor refinamiento; es decir, se supone que por no ser precisamente un misterio evolutivo, entonces no requiere una dilucidación de los mecanismos próximos —ontogenéticos- que la generan. En el segundo caso, hay de nuevo una ceguera ante la ontogenia porque se asume que la explicación evolutiva ha dado cuenta de lo más importante: el programa genético.

Es decir, parecería que parte del problema que subyace a la biología humana que versa sobre el "género" y la "sexualidad" —y nótese el entrecomillado - radica justamente en el olvido de la dimensión ontogenética. Fox Keller (1997) había anticipado ya que la biología 
del desarrollo tenía que concebirse como una ciencia feminista porque era en esta dimensión temporal donde se daba gran parte de la crítica feminista acerca de la importancia del contexto de crianza.

Esta intuición no es una excentricidad de dicha autora. Joan Roughgarden ha sostenido que resulta fundamental separar las explicaciones de la conducta animal en dos dimensiones temporales: una dimensión evolutiva que tradicionalmente se ha modelizado con genética de poblaciones, por un lado, y, por otro, una dimensión ontogenética que debe modelizarse por medio de teoría de juegos (Roughgarden 2004, 2009). Con esto ella rompe la tradición inaugurada por Maynard-Smith en la que para este último autor la teoría de juegos y la genética de poblaciones estaban modelizando una misma dimensión temporal. Pero el punto de Roughgarden se centra en enfatizar que hay dos dimensiones temporales a las que hay que distinguir claramente y que deben ser modelizadas de manera independiente en aquellas especies altriciales —es decir, que exhiben procesos de aprendizaje que van modificando sus conductas - cuya vida social exige cierta plasticidad conductual.

Importantes teóricos de la evolución ya habían anticipado estas dos ideas. Por un lado, Sober y Wilson (1998) habían enfatizado la necesidad de distinguir entre las explicaciones evolutivas del egoísmo (selfishness) y las explicaciones psicológicas sobre los motivos para las conductas egoístas (egoism); evidentemente estos autores estaban centrados en el llamado problema del altruismo, pero la sugerencia que subyace es básicamente la misma: distinguir entre un tiempo evolutivo y entre una dimensión psíquica motivacional. Por otro lado, el reconocimiento de la plasticidad de las conductas y la necesidad de tomar esto en cuenta a la hora de ofrecer una explicación evolutiva es algo que ya West-Eberhard (2003) había dicho.

En ese sentido, no es sólo el canon feminista al interior de la biología evolutiva el que ha sostenido que no debemos pasar por alto la dimensión ontogenética de la conducta. Ahora bien, en esta sección quiero mostrar básicamente cómo la filosofía de la biología puede ayudarnos a esclarecer cuál es el fallo de las explicaciones con las cuales abrí esta sección. Para ello introduciré los conceptos de falacia ontogenética y de falacia filogenética (Hochman 2012) para después explorar el alcance de la crítica abierta por los defensores de una concepción algo más desarrollista. Atendamos pues al primer punto.

Hochman acuña el término falacia ontogenética para complementar así la noción de falacia filogenética, que Lickliter y Berry (1990) 
originalmente postularon como una crítica a la psicología evolutiva. La manera en la que Hochman define ambas falacias depende de la distinción entre las explicaciones comparativas y las explicaciones contrastantes de Jackson y Petite (1992). En el caso de las explicaciones comparativas nuestro interés se centra ya sea en describir procesos robustos que pueden producir el mismo resultado incluso si las condiciones iniciales son algo diferentes o, usando la jerga de los mundos posibles, en describir procesos presentes en diversos mundos con configuraciones varias y que, sin embargo, exhiben dinámicas más o menos semejantes. Por otro lado, las explicaciones contrastantes recalcan justamente las diferencias en las trayectorias causales específicas que han generado cierto resultado o, de nuevo en la jerga de los mundos posibles, las diferencias de grano fino sobre cómo cierto proceso sucede en dos o más mundos. ${ }^{6}$

Sea como fuere, la distinción parece recuperar un asunto importante porque no es lo mismo describir la trayectoria específica de un proceso a describir el producto al que arriba más allá de la trayectoria que lo produjo. La diferencia radica en que la explicación comparativa es múltiplemente realizable mas no así la explicación contrastante.

Ahora bien, la falacia filogenética consiste precisamente en creer que una explicación comparativa da cuenta de la trayectoria específica del proceso a ser explicado, esto es, en suponer que una explicación evolutiva en términos de selección natural es una explicación acabada y que de cierta forma hace dispensable una explicación ontogenética.

En contraparte, la falacia ontogenética consiste en suponer que una explicación contrastante pone en evidencia a aquellos procesos robustos que están involucrados en la generación de una trayectoria específica, es decir, radica en asumir que una explicación de la tra-

${ }^{6}$ Para Hochman la analogía con las explicaciones evolutivas, por un lado, y las explicaciones ontogenéticas, por otro, le resulta lo suficientemente evidente como para proponer que las explicaciones evolutivas son comparativas y que las explicaciones ontogenéticas son contrastantes. A mi modo de ver, dicha equiparación resulta algo prematura puesto que las explicaciones evolutivas comúnmente se dividen en explicaciones evolutivas del patrón y en explicaciones evolutivas del proceso (Hull 1988). Quizá sólo las explicaciones evolutivas del proceso —y sólo en la medida en que se invoque a la selección natural o, quizá, a ciertas formas de autoorganizaciónson concebibles como explicaciones comparativas. En todo caso, las explicaciones evolutivas del patrón, las cuales se enfocan en la historia evolutiva de un linaje particular, son claramente ejemplos de explicaciones contrastantes. Algo parecido ocurrirá, muy probablemente, si atendemos a la ontogenia desde el punto de vista de los constreñimientos (explicaciones comparativas) o de la trayectoria ontogenética (explicación contrastante). 
yectoria ontogenética es una explicación completa. Nótese que esta objeción se aplica a toda explicación mecanicista que dé cuenta de la trayectoria de cada molécula en un proceso causal como los que Salmon (1990) definía como descripciones alternas — y mucho más exhaustivas - a las brindadas por la biología evolutiva.

En cierto sentido, la propuesta de Hochman es un llamado a la complementariedad de explicaciones que, si bien se asemeja a propuestas clásicas como la de Tinbergen (1963), ya no subraya las preguntas que se hacen sino aquello que una explicación enfatiza: robustez vs. descripciones detalladas.

Volviendo a la sinergia que es el eje de este texto, la nomenclatura que hasta aquí se ha ofrecido es justamente la que nos permite diagnosticar las fallas de las explicaciones con las que abrimos esta sección. Considerar, por ejemplo, que la heterosexualidad se explique porque - suponemos - es explicable en términos seleccionistas es cometer un claro ejemplo de la falacia filogenética; lo cierto es que seguimos sin conocer la trayectoria ontogenética que la genera. De igual manera, suponer que los modelos y narrativas en torno a la selección sexual nos permiten afirmar con certeza que los papeles de género vienen "precableados", o son innatos o heredables es otro ejemplo de una falacia filogenética en la cual se guarda un silencio estratégicamente conveniente para ocultar nuestra ignorancia sobre los mecanismos específicos que, creemos, deberían producir dichas conductas.

Algo parecido sucede cuando se ofrecen explicaciones sobre la homosexualidad, la bisexualidad o el transgenerismo y se enfatizan posibles alteraciones en mecanismos del desarrollo para luego dar un salto argumental y ofrecerlo como una explicación del porqué de la pervivencia de dichas conductas en términos evolutivos. En todos estos casos se ha cometido una falacia ontogenética.

Ahora bien, si nos enfocamos no tanto en la epistemología sino más bien en la agnotología (Proctor y Schiebinger 2008), es decir, en la así llamada epistemología de la ignorancia y su estudio de los procesos agnogenéticos - i.e., procesos de generación de ignorancia-, lo que observamos es que un análisis de la estructura explicativa a la luz de dichas falacias nos ayuda a comprender cómo se gestan procesos de naturalización y exclusión asociados a la generación de un conocimiento sobre la "naturaleza humana".

Para mayor precisión, la falacia filogenética que acompaña a la presunción de naturalidad de la heterosexualidad y la estructura tradicional de los papeles de género esconde la existencia de dos procesos agnogenéticos. Por un lado, oculta la asimetría en términos de 
las demandas de explicación que se le exigen a las identidades sexogenéricas disidentes en oposición a los roles de género más tradicionales. Así, la heterosexualidad y las identidades de género tradicionales se consideran ya explicadas en función de una naturaleza humana que la evolución ha forjado $\mathrm{y}$, por ende, no hay necesidad de un estudio de los mecanismos ontogenéticos que las generan. Esto es un proceso que genera ignorancia porque oculta la necesidad de investigar qué procesos biológicos o psíquicos participan en la generación y estabilización de dichos papeles e identidades.

Por otro lado, en un segundo proceso agnogenético se genera una injusticia hermenéutica (Fricker 2007) para todos aquellos miembros de las identidades sexo-genéricas disidentes a quienes se les considera como anomalías precisamente porque aparecen como los únicos sujetos a ser explicados. Esto no sólo genera procesos de exclusión y de intolerancia, también conduce a una resignificación de estos cuerpos que ya no son considerados sujetos a quien deba respetarse, sino objetos que deben ser intervenidos (ya sea para corregirlos o, al menos, para explicarlos) (Mc Manus 2012).

La filosofía de la biología y la filosofía de la ciencia pueden desempeñar aquí un papel importante. Por esta razón filósofas como Longino (2002) han considerado que es menester dejar atrás las discusiones sobre la axiología en las ciencias que se concentran únicamente en el problema que los valores idiosincrásicos le ocasionan a las presunciones de objetividad de la ciencia. Habría que tratar de incluir valores que desempeñen un papel mucho más positivo, valores como la inclusión y el cambio social que desde hace largo tiempo habitan los textos de las filosofías continentales, pero que sólo se asoman tímidamente en los trabajos de los filósofos analíticos de la biología.

\section{Conclusión: una agenda para el futuro}

Este texto es ante todo una invitación para que los filósofos de la biología y los estudiosos del género consideren la posibilidad de una interlocución en la cual ambos campos se enriquezcan. Esto es importante para evitar que el combate a un biologicismo desaforado se traduzca en una actitud anticientífica que termine por mirar con recelo al pensamiento evolutivo. Asimismo, es fundamental para evitar que la filosofía de la biología se convierta en una disciplina que habite una torre de marfil sin percatarse de los potenciales que tiene su propio aparato analítico. Y es que la filosofía de la biología justo podría servir de puente entre aquellos biólogos y biomédicos que buscan con entusiasmo dar cuenta del género y la sexualidad en términos 
biológicos, por un lado, y aquellos que miran esta aproximación con escepticismo y preocupación, por otro.

En ese sentido he tratado de mostrar algunas zonas de contacto, de las cuales he elaborado dos con más detenimiento. Primero, la discusión en torno al esencialismo y en qué forma la filosofía de la biología puede desempeñarse como una disciplina capaz de explicar qué se ha entendido por esencialismo en biología y qué se ha entendido por esencialismo en estudios de género. Sin duda la biología no es compatible con esencias aristotélicas o platónicas, lo cual todavía permite esencialismos de corte metodológico. Ocuparse de este punto permite esclarecer porque una biología evolutiva es todavía criticable por medio del argumento antiesencialista.

Segundo, he buscado ejemplificar cómo la falacia ontogenética y la falacia filogenética pueden esclarecer los argumentos esgrimidos por aquellos que consideran que se naturalizan ciertas categorías mientras que se marginalizan otras en el proceso mismo de postularlas como consecuencias o anomalías de la teoría evolutiva. En este sentido, tanto la filosofía de la explicación como la epistemología de la ignorancia resultan aliados valiosos para la crítica de género.

Queda así para el futuro y a modo de agenda la posibilidad de articular de una forma más detallada lo que Wilson (2005) denominó Constructivismo Social Evolutivo. Esto se debe a dos razones. Primero, porque se ha empleado como un modelo capaz de combinar las intuiciones de los constructivistas sociales que enfatizan la importancia del contexto histórico-social, y porque las intuiciones de los biólogos evolutivos que constantemente señalan que no podemos suponer que nuestros procesos cognitivos en sentido lato son una tabula rasa (Poiani 2010, Guerrero Mc Manus 2013). Segundo, porque a diferencia de otros modelos que buscan dar cuenta de la coevolución entre la cultura y lo biológico, el modelo de Wilson no es únicamente un modelo de coevolución genes-cultura, también anticipa perspectivas como la construcción de nicho y la importancia de un nivel de herencia simbólico (Jablonka y Lamb 2005) que no consistiría en explicar el origen del lenguaje, sino cómo éste ha hecho posible las narrativas y cómo éstas han modificado las conductas humanas.

Ahora bien, ninguna agenda a futuro estaría completa si pasamos por alto que la motivación de gran parte de los estudios de género y sexualidad tiene como objetivo la movilización de aparatos teóricos que se opongan a las injusticias cometidas en contra de mujeres y de otras minorías. Sin duda la filosofía de la biología tendría mucho que decir en este apartado. Hasta ahora la influencia del posestructuralismo francés se ha traducido en la defensa de las minorías utilizando 
discursos que enfatizan la otredad radical y el infierno que subyace en la pretensión humanista de afirmarnos como miembros de una misma humanidad (Baudrillard 1991, Latour 2002). Habrá que evaluar si este énfasis es conveniente o si puede y debe coexistir y complementarse con propuestas pertenecientes a la filosofía analítica que socavan la posibilidad de un relativismo radical (e.g., Davidson 1992). Por supuesto, ningún encuentro deja intactos a sus participantes; un encuentro entre estas disciplinas seguramente no será la excepción aunque al menos yo anticipo sinergías fecundas.

\section{BIBLIOGRAFÍA}

Bagemihl, B., 1999, Biological Exuberance: Animal Homosexuality and Natural Diversity, St. Martin's Press, Nueva York.

Baudrillard, J., 1991, La transparencia del mal. Ensayo sobre los fenómenos extremos, Anagrama, Barcelona.

Boyd, R., 1999, "Homeostasis, Species and Higher Taxa", en R.A. Wilson (ed.), Species, New Interdisciplinary Essays, The MIT Press, Boston.

Butler, J., 1993, Bodies that Matter: On the Discursive Limits of "Sex", Routledge, Nueva York.

Chomsky, N. y M. Foucault, 2006, La naturaleza humana. Justicia vs. poder, Katz Editores, Buenos Aires.

Davidson, D., 1992, Mente, mundo y acción, Paidós, México.

DeLamater, J., y H.J. Shibley, 1998, "Essentialism vs. Social Constructionism in the Study of Human Sexuality", The Journal of Sex Research, vol. 35, no. 1, pp. 10-18. doi:10.1080/00224499809551913 [fecha de última consulta: 12/04/2014].

Dupré, J., 2002, Humans and Other Animals, Oxford University Press, Oxford.

— 1993, The Disorder of Things. Metaphysical Foundations of the Disunity of Science, Harvard University Press, Boston.

Escobar, M.R., 2012, "Entre barroco y queer. El cuerpo trans en resistencia", en Parrini 2012, pp. 339-366.

Fausto-Sterling, A., 2000, Sexing the Body: Gender Politics and the Construction of Sexuality, Basic Books, Nueva York.

Federeci, S., 2004, Calibán y la bruja. Cuerpo, mujeres y acumulación originaria, Traficantes de Sueños, Madrid.

Foucault, M., 1977, Historia de la sexualidad. La voluntad de saber, Siglo XXI Editores, México.

*Agradezco los comentarios de los revisores anónimos cuyas críticas mejoraron tanto los contenidos como la legibilidad de este texto. Asimismo, quiero agradecer el apoyo de los diversos grupos de filosofía de la biología de la UNAM y la UAM-C por los espacios de interlocución que me han brindado. 
Fricker, M., 2007, Epistemic Injustice: Power and the Ethics of Knowing, Oxford University Press, Nueva York.

Friedman, M., 2000, A Parting of the Ways. Carnap, Cassirer, and Heidegger, Open Court, Chicago.

Garry, A., 2012, Analytic Feminism. The Stanford Encyclopedia of Philosophy, <http://plato.stanford.edu/archives/sum2012/entries/femapproachanalytic/ $>$ [fecha de última consulta: 12/04/2014].

Ghiselin, M., 1997, Metaphysics and the Origin of Species, State University of New York Press, Albany.

Guerrero Mc Manus, F., 2013, ¿Naces o te haces? La ciencia detrás de la homosexualidad, Paidós, México.

—_, 2012, "Las bases neuroendocrinas de la homosexualidad y la atomización mecanística del cuerpo", en Parrini 2012, pp. 173-210.

Haraway, D., 1989, Primate Visions: Gender, Race, and Nature in the World of Modern Science, Routledge, Chapman and Hall, Nueva York.

Hochman, A., 2012, "The Phylogeny Fallacy and the Ontogeny Fallacy", Biology and Philosophy, vol. 28, no. 4, pp. 593-612. doi: 10.1007/s10539-012-9325-3 [fecha de última consulta: 12/04/2014].

Hrdy, S.B., 1999, Mother Nature. Maternal Instincts and How They Shape the Human Species, Ballentine Books, Nueva York.

Hull, D., 1988, Science as a Process. An Evolutionary Account of the Social and Conceptual Development of Science, The University of Chicago Press, Chicago.

- 1965, "The Effect of Essentialism on Taxonomy: Two Thousand Years of Stasis I", British Journal for the Philosophy of Science, vol. 15, no. 60, pp. 314-326. doi: 10.1093/bjps/XV.60.314 [fecha de última consulta: 12/04/2014].

Irigaray, L., 1985, Speculum of the Other Woman, Cornell University Press, Ithaca.

Jablonka, E. y M. Lamb, 2005, Evolution in Four Dimensions: Genetic, Epigenetic, Behavioral, and Symbolic Variation in the History of Life, The MIT Press, Boston.

Jackson F. y P. Pettit, 1992, "In Defense of Explanatory Ecumenism", Economics and Philosophy, vol. 8, no. 1, pp. 1-21.

Keller, E.F., 1997, "Developmental Biology as a Feminist Cause?", Osiris, vol. 12, pp. 16-28.

Kristeva, J., 1982, Powers of Horror. An Essay of Abjection, Columbia University Press, Nueva York.

Latour, B., 2002, War of the Worlds. What about Peace?, Prickly Paradigm Press, Chicago.

Lickliter R. y T.D. Berry, 1990, “The Phylogeny Fallacy: Developmental Psychology's Misapplication of Evolutionary Theory", Developmental Review, vol. 10, pp. 348-364.

Lloyd, E., 2005, The Case of the Female Orgasm. Bias in the Science of Evolution, Harvard University Press, Boston. 
Longino, E.H., 2002, The Fate of Knowledge, Princeton University Press, Princeton.

Mc Manus, F., 2012, "The Structure of Explanations and CounterExplanations of Homosexuality", The Open Journal of Philosophy, vol. 2, no. 4, pp. 235-243. doi: 10.4236/ojpp.2012.24035 [fecha de última consulta: $12 / 04 / 2014]$.

—_, 2009, "Rational Disagreements in Phylogenetics", Acta Biotheoretica, vol. 57, pp. 99-127. doi: 10.1007/s10441-009-9072-2 [fecha de última consulta: 12/04/2014].

Navarro Reyes, J., 2010, Cómo hacer filosofía con palabras. A propósito del desencuentro entre Searle y Derrida, Fondo de Cultura Económica, México.

Nussbaum, M.C., 2012, Crear capacidades. Propuesta para el desarrollo humano, Paidós Estado y Sociedad, México.

— 2010 , From Disgust to Humanity. Sexual Orientation and Constitutional Law, Oxford University Press, Oxford.

— 1999, "The Professor of Parody", The New Republic, Febrero 22.

Oyama, S., 2000, Evolution's Eye. A Systems View of the Biology-Culture Divide, Duke University Press, Durham.

Parrini, R. (comp.), Los archivos del cuerpo. ¿Cómo estudiar el cuerpo?, PUEg-unam, México.

Poiani, A., 2010, Animal Homosexuality: A Biosocial Perspective, Cambridge University Press, Cambridge.

Preciado, B., 2002, Manifiesto contra-sexual, Opera Prima, Madrid.

Prieur, A., 2008, La casa de la mema: travestis, locas y machos, PUEGunam, México.

Proctor, R. y L. Schiebinger, 2008, Agnotology. The Making and Unmaking of Ignorance, Stanford University Press, Stanford.

Rieppel, O., 2009, "Species as a Process", Acta Biotheoretica, vol. 57, pp. 33-49.

Roughgarden, J., 2009, The Genial Gene: Deconstructing Darwinian Selfishness, Cooperation and the Evolution of Sex, University of California Press, Los Ángeles.

- 2004, Evolution's Rainbow: Diversity, Gender, and Sexuality in Nature and People, University of California Press, Los Ángeles.

Salmon, W., 1990, "Scientific Explanation. Causation and Unification", Crítica. Revista Hispanoamericana de Filosofía, vol. 22, no. 66, pp. 323.

Sober, E. y D.S. Wilson, 1998, Unto Others: The Evolution of Psychology and Unselfish Behavior, Harvard University Press, Cambridge.

Tinbergen, N., 1963, "On the Aims and Methods of Ethology", Zietschrift für Tierpsychologie, vol. 20, pp. 410-433.

West-Eberhard, M.J., 2003, Developmental Plasticity and Evolution, Oxford University Press, Nueva York. 
Wilson, D.S., 2005, "Evolutionary Social Constructivism", en J. Gottschall y D.S. Wilson (comps.), The Literary Animal: Evolution and the Nature of Narrative (Rethinking Theory), Northwestern University Press, Nueva York.

Zuk, M., 2002, Sexual Selection. What We Can and Can't Learn about Sex from Animals, University of California Press, Berkeley.

Recibido el 28 de junio de 2013; revisado el 13 de abril de 2014; aceptado el 13 de mayo de 2014. 